\title{
Awareness and recall during general anesthesia
}

\author{
Hyun Sik Chung
}

Department of Anaesthesiology and Pain Medicine, Seoul St. Mary's Hospital, The Catholic University of Korea, College of Medicine, Seoul, Korea

Anesthesia awareness is defined as both consciousness and recall of surgical events. New research has been conducted out to test this phenomenon. However, testing methods have not proven reliable, including those using devices based on electroencephalographic techniques to detect and prevent intraoperative awareness. The limitations of a standard intraoperative brain monitor reflect our insufficient understanding of consciousness. Moreover, patients who experience an intraoperative awareness can develop serious post-traumatic stress disorders that should not be overlooked. In this review, we introduce the incidence of intraoperative awareness during general anesthesia and discuss the mechanisms of consciousness, as well as risk factors, various monitoring methods, outcome and prevention of intraoperative awareness. (Korean J Anesthesiol 2014; 66: 339-345)

Key Words: Anesthesia, Complications, Electroencephalography, Intraoperative awareness, Post-traumatic stress disorders.

\section{Introduction}

Anesthesia awareness, also called unintended intraoperative awareness, is defined as the unintended experience and explicit recall of intraoperative events. The incidence of intraoperative awareness was reported to be $1.2 \%$ in 1960 [1]. Recent studies showed the incidence to be $0.1-0.2 \%$ in low-risk surgical procedures [2,3]; however, it can reach $1 \%$ for patients at increased risk $[4,5]$. General anesthesia was administered to 7 million patients in 2012 in the South Korea; therefore, intraoperative awareness can be estimated to occur in 7,000-14,000 cases [6].

Unintended intraoperative awareness is a potentially psychologically devastating complication of anesthesia associated with a high incidence of psychological sequelae, with post-traumatic stress disorders (PTSDs) being the most severe [7,8]. Intraoperative awareness is a serious iatrogenic complication causing high public concern, increases patients' apprehension of surgery and leads to medical-legal issues regarding anesthesia. In this review, unlike the connotation in cognitive science, the meaning of awareness implies both consciousness and explicit recall of the intraoperative period. As such, our use of awareness in this article implies explicit recall. Herein, we discuss the mechanisms of awareness and the clinical aspects of intraoperative awareness in adult patients, including risk factors, monitoring methods and prevention.

Received: January 27, 2014. Revised: April 7, 2014. Accepted: April 8, 2014.

Corresponding author: Hyun Sik Chung, M.D., Department of Anaesthesiology and Pain Medicine, Seoul St. Mary's Hospital, The Catholic University of Korea, College of Medicine, 505, Banpo-dong, Seocho-gu, Seoul 137-040, Korea. Tel: 82-2-2258-2236, Fax: 82-2-537-1951, E-mail: anesthe@catholic.ac.kr

(c) This is an open-access article distributed under the terms of the Creative Commons Attribution Non-Commercial License (http:// creativecommons.org/licenses/by-nc/3.0/), which permits unrestricted non-commercial use, distribution, and reproduction in any medium, provided the original work is properly cited. 


\section{Mechanisms of Awareness}

The current fundamental limitation of clinical practice is a lack of ability to distinguish between the anesthetized and the awakened. The brain is a major target organ of general anesthesia; however, reliable methodologies for standard monitoring of drug action on brain function have not been elucidated. Current intraoperative monitors estimate the side effects rather than the primary effects of general anesthesia. The lack of standard cerebral function monitor under general anesthesia is considered insufficient understanding the effects of anesthetic drugs on the brain. Therefore, better understanding of the neurobiology mechanisms regarding awareness is important for improving the monitoring of intraoperative awareness.

A description of mechanisms classically begins at the molecular level; however, consciousness mechanisms result from complex interactions in the brain. Thus, explaining how the brain is aroused and how drugs used for general anesthesia modulate this process should be based on the sleep-wake mechanisms. Although sleep and anesthesia are substantially different phenomena, they share phenotypic characteristics and underlying mechanisms. The hypothesis that anesthetic drugs act via subcortical sleep centers was published in the mid-1990s [9], and further supported with empirical data [10,11]. Normal sleep-wake cycles are regulated by several nuclei located in the pons, midbrain, hypothalamus, and basal forebrain. These centers generally are de-active during sleep; on the other hand, other centers such as the ventrolateral preoptic nucleus are active. This phenomenon can be explained by the wake-on/sleepoff and sleep-no/wake-off nuclei that are thought to inhibit each other reciprocally, leading to the hypothesis of a flip-flop sleepwake mechanism [12-14]. For example, the noradrenergic locus ceruleus in the pons and the histaminergic tuberomammillary nucleus in the posterior hypothalamus are active during wakefulness, whereas the $\gamma$-aminobutyric acid-transmitting (GABAergic) ventrolateral preoptic nucleus is inhibited. Similarly, the ventrolateral preoptic nucleus becomes active during sleep and then inhibits the activity of the arousal-promoting locus ceruleus and tuberomammillary nuclei.

Anesthetic and sedative drugs act by modulating the activity of these structures. For example, the hypnotic effects of dexmedetomidine are associated with the activation of $\alpha_{2}$-adrenergic receptors and inhibition of noradrenergic projections from the locus ceruleus [15]. The presence of electroencephalographic (EEG) sleep spindles during halothane anesthesia is associated with a reduction of cholinergic transmission from the pedunculopontine and laterodorsal tegmentum [16]. Other drugs, such as propofol or isoflurane may exert hypnotic effects by inhibiting or interrupting histaminergic transmission to the tuberomammillary nucleus $[17,18]$. The arousal-promoting orexinergic neu- rons in the hypothalamus are thought to play an essential role in the emergence from sevoflurane and isoflurane anesthesia [19], but not from halothane anesthesia [20]. This variability suggests that the effects of general anesthetic drugs on sleep-wake centers are specific to individual agents, meaning anesthetic and sedative drugs have agent specific effects. The ventro-lateral preoptic nucleus in the inhibitory center is activated by GABAergic drugs (e.g., propofol) and not by the N-methyl-D-aspartate glutamate receptor antagonist (e.g., ketamine) [18,21]. Additionally, on the behavioral level, various anesthetic drugs have differential effects on the sleep-regulating pathways [22].

\section{Risk Factor}

Risk factors for intraoperative awareness include multiple traumas, cardiac surgery, emergency cesarean section delivery, difficult intubation and patients with a previous history of awareness. According to epidemiological studies, risk factors can be classified into three subgroups: patients, surgical procedures, and anesthetic techniques.

The incidence of intraoperative awareness is threefold higher in females than males and females recover more rapidly than males form anesthesia [23,24]. Younger patients $(<60$ years of age) showed a higher incidence of intraoperative awareness [2,25]; however, Pollard et al. [26] reported a higher incidence in elderly patients. The incidence of awareness in children increased to $0.8 \%$ [4,27]. Patients with addiction require a greater amount of anesthetic drugs; thus, those patients have an increased likelihood of experiencing intraoperative awareness $[28,29]$. A previous history of awareness is a strong predisposing factor that increases the incidence of a new intraoperative awareness to $1.6 \%$ [30]. In patients with difficulty airways, the incidence of intraoperative awareness is 4.5-7.5\% [31]. Reportedly, the incidence of anesthesia awareness is greater in patients who require a lower dose of general anesthetic drug, which is thus titrated to reduce the probability of serious side effects according to the patient's physical condition; for example, a patient who is unstable hemodynamically, has hypothermia, or acute intoxication [28,32]. Surgical procedures typically identified as belonging to this category are cardiac, obstetric and multiple trauma surgeries [28]. Patients undergoing cardiopulmonary bypass have reported an awareness incidence from 1.1-23\% $[33,34]$. The incidence of awareness related to obstetric anesthesia ranges from $0.4-1.3 \%[29,35,36]$. Factors associated with obstetric anesthesia are rapid sequence induction and a reduced inhaled fraction of inhalant anesthetics [37]. Other risk factors for anesthesia awareness include the use of intravenous anesthesia as opposed to inhalant anesthetics and the premature lightening of anesthesia at the end of surgery to facilitate surgical turnover. Furthermore, the estimated anesthetic concentration 
in target organs informed by a target-controlled infusion system is not the real-time concentration of anesthetic in the patient during intravenous anesthesia. The use of neuromuscular blocking agents is associated with a higher incidence of intraoperative awareness $(0.18 \%$ vs $0.1 \%)$ [3]. The administration of neuromuscular blocking agents may impede patient's movements which are a more useful and simpler method of detecting perioperative awareness [38,39].

\section{Awareness Experience}

Patients experience awareness differently and have described auditory recalls (48\%), feelings of difficult breathing (48\%) and painful sensations (28\%) [2]. Some patients state that occurrence of intraoperative awareness is their worst hospital experience and others are not willing to undergo surgery or anesthesia again. Patients commonly reported hearing intraoperative sounds or conversations. Other common experiences include pain sensation, being touched, fear, visual perceptions and feeling helpless.

\section{Detection}

Preventing intraoperative awareness in patients undergoing general anesthesia can be challenging for an anesthesiologist. Although monitoring methods are available, recognition of intraoperative awareness is difficult. Intraoperative awareness cannot be detected during general anesthesia, because the awareness recall can be identified only postoperatively by obtaining information directly from the patient. To date, no reliable methodology exists due to the limitations in detecting awareness and an insufficient understanding of consciousness. Moreover, conventional indicators of physiological and motor responses, such as high blood pressure, rapid heart rate, or movement are often masked by the neuromuscular blocking agents used to achieve the necessary muscle relaxation during surgery, as well as the concurrent administration of other drugs such as beta-blockers or calciumchannel blockers.

To overcome the limitations of existing methods, new techniques of detecting intraoperative awareness that are less affected by the drugs are being developed. These devices focus on measuring brain activity rather than physiological responses; these EEG devices include the Bispectral Index ${ }^{\circledR}$ (BIS; Aspect Medical Systems, Natick, MA, USA), which are designed to prevent and detect intraoperative awareness in the high-risk patients. Evidence that these devices detect and prevent intraoperative awareness is lacking. However, regarding BIS monitoring, the US Food and Drug Administration stated that "Use of BIS monitoring to help guide anesthetic administration may be associated with the reduction of the incidence of awareness with recall in adults during general anesthesia and sedation."

In the present review, the ability to detect and prevent intraoperative awareness was estimated using a prediction probability $(\mathrm{Pk})$ value. A Pk value resulting from correlational measurement provides a measure of how well a monitor or technique can differentiate between two different clinical states [40]. A Pk value of 1.0 indicates a perfect association and a Pk value of 0.50 a $50: 50$ probability.

\section{Clinical examinations and conventional monitoring}

The clinical examinations used to evaluate intraoperative awareness include watching for movements, response to commands, eyes opening, eyelash reflexes, pupil responses or diameters, tearing and perspiration. Conventional monitoring systems include vital signs, such as blood pressure (BP) and heart rate (HR) as well as end-tidal anesthetic gas analyzer. $\mathrm{Pk}$ values ranged from $0.74-0.76$ for the association between reflex or movement and depth of anesthesia [40]. One study reported a significant association between response to command and memory when propofol was administered during induction of general anesthesia [41]. Among conventional monitoring systems, $\mathrm{Pk}$ values for mean arterial pressure (MAP) ranged from $0.68-0.94$ between a responsive and unresponsive state and HR $\mathrm{Pk}$ values ranged from $0.50-0.82$ [42-44]. However, wide ranges in MAP and HR values were observed during the intraoperative period and awareness was reported in the absence of tachycardia or hypertension [45].

Clinical examinations (purposeful and reflex movements) and conventional monitoring systems (electrocardiogram, BP, $\mathrm{HR}$, end-tidal anesthetic gas concentration and capnography) are beneficial and should be used for assessment of intraoperative awareness.

\section{Brain electrical activity monitoring}

Most devices that brain electrical activity for evaluating anesthetic effects are based on EEG activity using electrodes places on the forehead and use an index of $0-100$. This index demonstrates the clinical states of consciousness. The value of 100 indicates the awaken state and the 0 value an isoelectric EEG (absence of brain electrical activity) as deep anesthesia [46]. These devices also provide an electromyographic (EMG) activity for artifact recognition. Although an EMG activity obtained from scalp muscles, it is an important source of clinically significant information. Sudden appearance of frontal EMG activity implies a somatic response to noxious stimulation due to inadequate analgesia, therefore providing a potential warning of impending awareness. For this reason, several monitors of brain electrical activity provide information on the level of EMG activity. 


\section{Spontaneous EEG monitors}

\section{Bispectral Index}

The BIS calculates an index using a proprietary algorithm based on a single channel of frontal EEG activity. The algorithm calculates an index using EEG time domain (burst-suppression analysis) and frequency domain (power spectrum). This index is scaled between 0 (isoelectrical activity) and 100 (awakened state), indicating the hypnotic level. Specific ranges (40-60) were recommended to reflect a low probability of awareness with recall during general anesthesia. The BIS values are empirically derived from a database of more than 1,500 anesthetic drugs prospectively and translate changes of nonlinear stages in EEG activity to a linear decrease in the BIS index with increasing plasma concentrations of various anesthetic drugs. One randomized controlled trial that enrolled 2,500 high-risk patients reported explicit recall in $0.91 \%$ of patients in routine clinical practice, whereas the incidence of awareness decreased significantly to $0.17 \%$ of patients when BIS monitors were applied [47]. Pk values using BIS ranged from $0.72-1.00$ between awake and loss of response, and from $0.79-0.97$ between an anesthetized state and first response [42-44,48-51].

\section{Entropy}

The Entropy (GE Healthcare Technologies, Waukesha, WI, USA) calculated the indices by measuring irregularity in the data acquisition of EEG and frontal EMG signals. The indices consist of two separate parameters; the fast-reacting Response Entropy (RE) and the more steady and robust State Entropy (SE). Highly irregular signals with varying wavelength and amplitude over time produce high entropy values and indicate that the patient is an awakened state. More ordered signals with less variation in wavelength and amplitude over time produce low or 0 entropy values, representing suppression of brain electrical activity and a low probability of awareness. SE ranges from 0 (no brain activity) to 91 (awake), computed over the frequency range $0.8-32 \mathrm{~Hz}$, reflecting the cortical state of the patient. RE ranges from 0 (no brain activity) to 100 (awake), computed over a frequency range of $0.8-47 \mathrm{~Hz}$ and contain EMG frequencies. Therefore, RE values respond to the EMG activity resulting from inadequate analgesia. SE values are always less than or equal to $\mathrm{RE}$, and based on the estimated hypnotic effect of anesthetic drugs during general anesthesia because they are based on the EEG signal. Several studies have reported the $\mathrm{Pk}$ values for loss of consciousness: RE, 0.83-0.97; SE, 0.81-0.90 [41,52,53].

\section{Narcotrend}

The Narcotrend ${ }^{\circledR}$ (MonitorTechnik, Bad Branstedt, Germany) analyzes the raw EEG data using spectral analysis to produce a number of parameters and provides an automatically classified
EEG using proprietary pattern recognition algorithms. The EEG classification scale is A (awake), B (sedated), C (light anesthesia), D (general anesthesia), E (general anesthesia with deep hypnosis) and $\mathrm{F}$ (general anesthesia with increasing burst suppression). The system included a series of subclassifications resulting in a total of 14 possible substages: A, B0-2, C0-2, D0-2, E0-1 and $\mathrm{F} 0-1$. The $\mathrm{A}-\mathrm{F}$ scale is also translated into a dimensionless index $(0=$ deep general anesthesia, $100=$ awake $)$ similar to the BIS index. Pk values ranged from $0.93-0.99$ between awake and loss of response and from $0.94-0.99$ between an anesthetized state and first response $[43,44]$.

\section{Patient State Analyzer}

The Patient State Index (PSI; Physiometrix, North Billerica, MA, USA) is derived from the processed four-channel EEG. The PSI is the result of an observation that reversible spatial changes occur in power distribution of quantitative EEG at loss and return of consciousness. The PSI ranges from 0-100 $(0=$ deep anesthesia, $100=$ awake) similar to BIS, entropy, and Narcotrend. One study reported a Pk value of 0.70 for predicting response to command, with a sensitivity of $85.6 \%$ and specificity of $38.8 \%$ [54]. Another study published a significant correlation between the PSI and unconsciousness [55].

\section{SNAP index}

The SNAPII (Everest Biomedical Instruments, Chesterfield, MO, USA) produces an index based on a single EEG channel derived from a spectral analysis of EEG activity based on lowfrequency $(0.1-18 \mathrm{~Hz})$, high-frequency $(80-420 \mathrm{~Hz})$ and a burst suppression algorithm. A mean SNAP index of 71 represents $95 \%$ of elective surgery patients with a loss of consciousness [56].

Cerebral State Monitor/Cerebral State Index

The Cerebral State Monitor (Danmeter A/S, Odense, Denmark) produces an index ( $0=$ isoelectrical activity, $100=$ awake $)$ by analyzing a single EEG channel. In addition, it provides EEG suppression percentage and a measure of EMG activity (75-85 $\mathrm{Hz}$ ). To date, No correlation between the Cerebral State Monitor and the incidence of intraoperative awareness has been reported.

\section{Evoked brain electrical activity monitor}

\section{AEP Monitor/2}

AEP Monitor/2 (Danmeter A/S, Odense, Denmark) extracts the auditory evoked potentials (AEPs) from the electrical responses (EEG-signal) of the brainstem by using the auditory radiation and the auditory cortex responding to auditory sound stimuli. The change in AEP for regarding the anesthetic drug effect has been investigated since the 1980s [57-59]. The brainstem response is less sensitive to anesthetic drugs, whereas middlelatency AEPs as early cortical responses respond predictably to 
alternating of concentrations of both volatile and intravenous anesthetic drugs. The AEP response with an increased concentration of anesthetic drug showed an increased latency and decreased amplitude. These AEP signals are necessary in signal averaging techniques because the AEP signal is extremely low $(<1 \mu \mathrm{V})$. The AEP index (AAI) is calculated using a mathematical analysis of the AEP waveform and is a correlation of drug effect of general anesthetics. The AAI ranges from 0-100 with an $\mathrm{AAI}>50$ corresponding to the awake state and ranges from 15-25 for anesthesia, lower numerical thresholds in contrast to other EEG indices. One study reported an awareness Pk value of 0.99 after laryngeal mask airway insertion [60].

\section{Outcomes}

The percentage of patients reported to experience mental distress, including an indeterminate number with PTSDs is 33-69\% $[8,61]$. The majority of these patients fear future surgery and anesthesia. An early emotional response after an intraoperative awareness experience is a risk factor for developing PTSDs [62].

\section{Prevention}

The risk of intraoperative awareness may be minimized using specific clinical practice principles.

\section{Preoperative evaluation}

A preoperative evaluation includes (1) obtaining a history (medical records, laboratory findings and patient-and-family interview), (2) performing a physical examination, (3) identifying patients' risk factors for intraoperative awareness (type of anesthesia and surgery) and (4) informing selected patients regarding the possibility of intraoperative awareness.

A preoperative evaluation also includes substance use or abuse, limited hemodynamic reserve, and ASA physical status of IV or V. A previous history of intraoperative awareness is a strong predisposing factor for intraoperative awareness.

\section{Preinduction of general anesthesia}

The preinduction phase of general anesthesia includes (1) inspecting the functioning of anesthesia delivery systems and (2) the prophylactic administration of benzodiazepines. Checking the functioning of anesthesia delivery systems is helpful to exclude drug errors and drug delivery errors [45,63-66]. Use of benzodiazepine should be limited to patients requiring smaller dosages of anesthetic drugs such as those undergoing cardiac surgery, emergency surgery and patients with multiple traumas and can be beneficial in patients undergoing cesarean delivery as well as total intravenous anesthesia.

\section{Intraoperative interventions}

Intraoperative interventions include (1) cautionary use of the neuromuscular blocking agents (applying a monitor for the neuromuscular function and maintaining T1 > 5\%), (2) inhalant anesthetics must be monitored with end-tidal gas analyzers and the minimum alveolar concentration (MAC) of anesthetic agents should be maintained $>0.8$ and (3) the BIS value $<60$ [29].

\section{Postoperative interventions}

Postoperative interventions include (1) conducting a structured postoperative interview with patients to define the nature of the episode if intraoperative awareness was reported, and (2) providing postoperative counseling or psychological support.

An explicit query of perioperative awareness should be performed to identify the occurrence of awareness. Physicians should specifically ask their patients regarding intraoperative awareness because patients usually do not confide in their physicians.

\section{Conclusion}

Anesthesia awareness can be experienced as a psychological trauma by patients undergoing general anesthesia; the psychological damage is unpredictable and immeasurable. Although the various EEG monitors are imperfect for detecting and preventing anesthesia awareness, attention to depth of anesthesia, appropriate use of anesthetic drugs and the judicious use of neuromuscular blocking agents should be strictly applied to patients undergoing general anesthesia. Various monitoring devices can be used to detect anesthesia awareness to minimize the incidence of this potentially devastating complication. Future work should include research and development of precise reliable detection systems, systems for real-time delivery and monitoring of intravenous anesthetic drugs, the identification of the appropriate lower MAC limit and the development of new drugs that target consciousness and memory while minimizing adverse effects. 


\section{References}

1. Hutchinson R. Awareness during surgery. A study of its incidence. Br J Anaesth 1961; 33: 463-9.

2. Sebel PS, Bowdle TA, Ghoneim MM, Rampil IJ, Padilla RE, Gan TJ, et al. The incidence of awareness during anesthesia: a multicenter United States study. Anesth Analg 2004; 99: 833-9.

3. Sandin RH, Enlund G, Samuelsson P, Lennmarken C. Awareness during anaesthesia: a prospective case study. Lancet 2000; 355: 707-11.

4. Davidson AJ, Huang GH, Czarnecki C, Gibson MA, Stewart SA, Jamsen K, et al. Awareness during anesthesia in children: a prospective cohort study. Anesth Analg 2005; 100: 653-61.

5. Lopez U1, Habre W, Laurençon M, Haller G, Van der Linden M, Iselin-Chaves IA. Intra-operative awareness in children: the value of an interview adapted to their cognitive abilities. Anaesthesia 2007; 62: 778-89.

6. Service HIRA. Available from http://www.hira.or.kr/rd/dissdic/infoMdfeeList.do?pgmid=HIRAA020044030000.

7. Leslie K, Chan MT, Myles PS, Forbes A, McCulloch TJ. Posttraumatic stress disorder in aware patients from the B-aware trial. Anesth Analg 2010; 110: 823-8.

8. Osterman JE, Hopper J, Heran WJ, Keane TM, van der Kolk BA. Awareness under anesthesia and the development of posttraumatic stress disorder. Gen Hosp Psychiatry 2001; 23: 198-204.

9. Lydic R, Biebuyck JF. Sleep neurobiology: relevance for mechanistic studies of anaesthesia. Br J Anaesth 1994; 72: 506-8.

10. Vanini G, Watson CJ, Lydic R, Baghdoyan HA. Gamma-aminobutyric acid-mediated neurotransmission in the pontine reticular formation modulates hypnosis, immobility, and breathing during isoflurane anesthesia. Anesthesiology 2008; 109: 978-88.

11. Orser BA, Saper CB. Multimodal anesthesia and systems neuroscience: the new frontier. Anesthesiology 2008; 109: 948-50.

12. Lu J, Sherman D, Devor M, Saper CB. A putative flip-flop switch for control of REM sleep. Nature 2006; 441: 589-94.

13. Saper CB, Scammell TE, Lu J. Hypothalamic regulation of sleep and circadian rhythms. Nature 2005; 437: 1257-63.

14. Saper CB, Chou TC, Scammell TE. The sleep switch: hypothalamic control of sleep and wakefulness. Trends Neurosci 2001; 24: 726-31.

15. Nelson LE, Lu J, Guo T, Saper CB, Franks NP, Maze M. The alpha2-adrenoceptor agonist dexmedetomidine converges on an endogenous sleep-promoting pathway to exert its sedative effects. Anesthesiology 2003; 98: 428-36.

16. Keifer JC, Baghdoyan HA, Lydic R. Pontine cholinergic mechanisms modulate the cortical electroencephalographic spindles of halothane anesthesia. Anesthesiology 1996; 84: 945-54.

17. Luo T, Leung LS. Basal forebrain histaminergic transmission modulates electroencephalographic activity and emergence from isoflurane anesthesia. Anesthesiology 2009; 111: 725-33.

18. Nelson LE, Guo TZ, Lu J, Saper CB, Franks NP, Maze M. The sedative component of anesthesia is mediated by GABA(A) receptors in an endogenous sleep pathway. Nat Neurosci 2002; 5: 979-84.

19. Kelz MB, Sun Y, Chen J, Cheng Meng Q, Moore JT, Veasey SC, et al. An essential role for orexins in emergence from general anesthesia. Proc Natl Acad Sci U S A 2008; 105: 1309-14.

20. Gompf H, Chen J, Sun Y, Yanagisawa M, Aston-Jones G, Kelz MB. Halothane-induced hypnosis is not accompanied by inactivation of orexinergic output in rodents. Anesthesiology 2009; 111: 1001-9.

21. Lu J, Nelson LE, Franks N, Maze M, Chamberlin NL, Saper CB. Role of endogenous sleep-wake and analgesic systems in anesthesia. J Comp Neurol 2008; 508: 648-62.

22. Mashour GA, Lipinski WJ, Matlen LB, Walker AJ, Turner AM, Schoen W, et al. Isoflurane anesthesia does not satisfy the homeostatic need for rapid eye movement sleep. Anesth Analg 2010; 110: 1283-9.

23. Ghoneim M. The trauma of awareness: history, clinical features, risk factors, and cost. Anesth Analg 2010; 110: 666-7.

24. Hoymork SC, Raeder J. Why do women wake up faster than men from propofol anaesthesia? Br J Anaesth 2005; 95: 627-33.

25. Mashour GA, Wang LY, Turner CR, Vandervest JC, Shanks A, Tremper KK. A retrospective study of intraoperative awareness with methodological implications. Anesth Analg 2009; 108: 521-6.

26. Pollard RJ, Coyle JP, Gilbert RL, Beck JE. Intraoperative awareness in a regional medical system: a review of 3 years' data. Anesthesiology 2007; 106: 269-74.

27. Blussé Van Oud-Alblas HJ, Bösenberg AT, Tibboel D. Awareness in children: another two cases. Paediatr Anaesth 2008; $18: 654-7$.

28. Ghoneim MM. Awareness during anesthesia. Anesthesiology 2000; 92: 597-602.

29. Ghoneim MM, Block RI. Learning and memory during general anesthesia: an update. Anesthesiology 1997; 87: 387-410.

30. Ghoneim MM, Block RI, Haffarnan M, Mathews MJ. Awareness during anesthesia: risk factors, causes and sequelae: a review of reported cases in the literature. Anesth Analg 2009; 108: 527-35.

31. Shiga T, Wajima Z, Inoue T, Sakamoto A. Predicting difficult intubation in apparently normal patients: a meta-analysis of bedside screening test performance. Anesthesiology 2005; 103: 429-37.

32. Bogetz MS, Katz JA. Recall of surgery for major trauma. Anesthesiology 1984; 61: 6-9.

33. Goldmann L, Shah MV, Hebden MW. Memory of cardiac anaesthesia. Psychological sequelae in cardiac patients of intra-operative suggestion and operating room conversation. Anaesthesia 1987; 42: 596-603.

34. Wang Y, Yue Y, Sun YH, Wu AS, Wu QW, Zhang YQ, et al. Investigation and analysis of incidence of awareness in patients undergoing cardiac surgery in Beijing, China. Chin Med J (Engl) 2005; 118: 1190-4. 
35. Lyons G, Macdonald R. Awareness during caesarean section. Anaesthesia 1991; 46: 62-4.

36. Paech MJ, Scott KL, Clavisi O, Chua S, McDonnell N. A prospective study of awareness and recall associated with general anaesthesia for caesarean section. Int J Obstet Anesth 2008; 17: 298-303.

37. Yeo SN, Lo WK. Bispectral index in assessment of adequacy of general anaesthesia for lower segment caesarean section. Anaesth Intensive Care 2002; 30: 36-40.

38. Kotsovolis G, Komninos G. Awareness during anesthesia: how sure can we be that the patient is sleeping indeed? Hippokratia 2009; 13: 83-9.

39. Myles PS. Prevention of awareness during anaesthesia. Best Pract Res Clin Anaesthesiol 2007; 21: 345-55.

40. Smith WD, Dutton RC, Smith NT. Measuring the performance of anesthetic depth indicators. Anesthesiology 1996; 84: 38-51.

41. Dutton RC, Smith WD, Smith NT. Wakeful response to command indicates memory potential during emergence from general anesthesia. J Clin Monit 1995; 11: 35-40.

42. Schmidt GN, Bischoff P, Standl T, Hellstern A, Teuber O, Schulte Esch J. Comparative evaluation of the Datex-Ohmeda S/5 Entropy Module and the Bispectral Index monitor during propofol-remifentanil anesthesia. Anesthesiology 2004; 101: 1283-90.

43. Schmidt GN, Bischoff P, Standl T, Jensen K, Voigt M, Schulte Am Esch J. Narcotrend and Bispectral Index monitor are superior to classic electroencephalographic parameters for the assessment of anesthetic states during propofol-remifentanil anesthesia. Anesthesiology 2003; 99: 1072-7.

44. Schmidt GN, Bischoff P, Standl T, Lankenau G, Hilbert M, Schulte Am Esch J. Comparative evaluation of Narcotrend, Bispectral Index, and classical electroencephalographic variables during induction, maintenance, and emergence of a propofol/remifentanil anesthesia. Anesth Analg 2004; 98: 1346-53.

45. Domino KB, Posner KL, Caplan RA, Cheney FW. Awareness during anesthesia: a closed claims analysis. Anesthesiology 1999; 90: 1053-61.

46. Rampil IJ. A primer for EEG signal processing in anesthesia. Anesthesiology 1998; 89: 980-1002.

47. Myles PS, Leslie K, McNeil J, Forbes A, Chan MT. Bispectral index monitoring to prevent awareness during anaesthesia: the B-Aware randomised controlled trial. Lancet 2004; 363: 1757-63.

48. Glass PS, Bloom M, Kearse L, Rosow C, Sebel P, Manberg P. Bispectral analysis measures sedation and memory effects of propofol, midazolam, isoflurane, and alfentanil in healthy volunteers. Anesthesiology 1997; 86: 836-47.

49. Schraag S, Bothner U, Gajraj R, Kenny GN, Georgieff M. The performance of electroencephalogram bispectral index and auditory evoked potential index to predict loss of consciousness during propofol infusion. Anesth Analg 1999; 89: 1311-5.

50. Kreuer S, Bruhn J, Larsen R, Bialas P, Wilhelm W. Comparability of Narcotrend index and bispectral index during propofol anaesthesia. Br J Anaesth 2004; 93: 235-40.

51. Kreuer S, Bruhn J, Larsen R, Hoepstein M, Wilhelm W. Comparison of Alaris AEP index and bispectral index during propofol-remifentanil anaesthesia. Br J Anaesth 2003; 91: 336-40.

52. Bruhn J, Bouillon TW, Radulescu L, Hoeft A, Bertaccini E, Shafer SL. Correlation of approximate entropy, bispectral index, and spectral edge frequency 95 (SEF95) with clinical signs of "anesthetic depth" during coadministration of propofol and remifentanil. Anesthesiology 2003; 98: 621-7.

53. Vanluchene AL, Struys MM, Heyse BE, Mortier EP. Spectral entropy measurement of patient responsiveness during propofol and remifentanil. A comparison with the bispectral index. Br J Anaesth 2004; 93: 645-54.

54. Schneider G, Gelb AW, Schmeller B, Tschakert R, Kochs E. Detection of awareness in surgical patients with EEG-based indices--bispectral index and patient state index. Br J Anaesth 2003; 91: 329-35.

55. Chen X, Tang J, White PF, Wender RH, Ma H, Sloninsky A, et al. A comparison of patient state index and bispectral index values during the perioperative period. Anesth Analg 2002; 95: 1669-74.

56. Wong CA, Fragen RJ, Fitzgerald PC, McCarthy RJ. The association between propofol-induced loss of consciousness and the SNAP index. Anesth Analg 2005; 100: 141-8.

57. Thornton C, Konieczko K, Jones JG, Jordan C, Doré CJ, Heneghan CP. Effect of surgical stimulation on the auditory evoked response. Br J Anaesth 1988; 60: 372-8.

58. Kenny GN, Mantzaridis H. Closed-loop control of propofol anaesthesia. Br J Anaesth 1999; 83: 223-8.

59. Schwender D, Conzen P, Klasing S, Finsterer U, Pöppel E, Peter K. The effects of anesthesia with increasing end-expiratory concentrations of sevoflurane on midlatency auditory evoked potentials. Anesth Analg 1995; 81: 817-22.

60. Doi M, Gajraj RJ, Mantzaridis H, Kenny GN. Prediction of movement at laryngeal mask airway insertion: comparison of auditory evoked potential index, bispectral index, spectral edge frequency and median frequency. Br J Anaesth 1999; 82: 203-7.

61. Lennmarken C, Sandin R. Neuromonitoring for awareness during surgery. Lancet 2004; 363: 1747-8.

62. Samuelsson P, Brudin L, Sandin RH. Late psychological symptoms after awareness among consecutively included surgical patients. Anesthesiology 2007; 106: 26-32.

63. Bergman IJ, Kluger MT, Short TG. Awareness during general anaesthesia: a review of 81 cases from the Anaesthetic Incident Monitoring Study. Anaesthesia 2002; 57: 549-56.

64. Caplan RA, Vistica MF, Posner KL, Cheney FW. Adverse anesthetic outcomes arising from gas delivery equipment: a closed claims analysis. Anesthesiology 1997; 87: 741-8.

65. Masuda A, Arai Y, Hirota K, Shibuya N, Ito Y. Misuse of infusion pump during propofol anaesthesia. Can J Anaesth 1998; 45 : 187.

66. Tong D, Chung F. Recall after total intravenous anaesthesia due to an equipment misuse. Can J Anaesth 1997; 44: 73-7. 